Cahiers de philosophie de l'université de

49 | 2012

Levinas : au-delà du visible

\title{
Le monde cassé et le moi comme exil
}

\section{Emmanuel Housset}

\section{(2) OpenEdition}

Journals

Édition électronique

URL : https://journals.openedition.org/cpuc/882

DOI : $10.4000 /$ cpuc.882

ISSN : 2677-6529

\section{Éditeur}

Presses universitaires de Caen

\section{Édition imprimée}

Date de publication : 31 juillet 2012

Pagination : 229-252

ISBN : 978-2-84133-410-0

ISSN : 1282-6545

Référence électronique

Emmanuel Housset, "Le monde cassé et le moi comme exil », Cahiers de philosophie de l'université de Caen [En ligne], 49 | 2012, mis en ligne le 07 juin 2018, consulté le 03 février 2023. URL : http:// journals.openedition.org/cpuc/882; DOI : https://doi.org/10.4000/cpuc.882

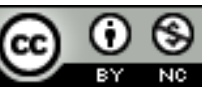

Creative Commons - Attribution - Pas d'Utilisation Commerciale 4.0 International - CC BY-NC 4.0 https://creativecommons.org/licenses/by-nc/4.0/ 


\section{Le monde cassé et le moi comme exil}

\footnotetext{
$\mathrm{E}$
}

MMANUEl Levinas donne une toute nouvelle dimension au thème ancien de l'exil du sage, et cela en parvenant à tenir ensemble les deux figures fondamentales de l'exil, à savoir l'exil comme privation du chez soi, comme relégation et déportation, et l'exil comme mouvement de transcendance, comme itinéraire de l'âme vers le non-lieu de l'amour. Néanmoins les deux volumes d'inédits marquent particulièrement à quel point cette compréhension de soi comme exil s'oppose radicalement à la conception stoïcienne de l'exil telle qu'il est possible de la trouver notamment dans les écrits de Sénèque. Certes la philosophie stoïcienne décrit la brutalité de l'arrachement à soi lié au fait de devoir quitter sa patrie, cependant un tel exil appartient pour les Stoïciens au cours des choses, et c'est pourquoi il importe de se comprendre comme un citoyen du monde qui est partout chez lui et pour lequel aucune terre n'est étrangère. L'exil ne serait alors qu'un élargissement du chez soi par lequel l'homme passe de sa patrie au monde entier comme lieu où il peut être. Dans cette métamorphose propre à l'exil, le lieu de l'exil importe peu, puisque la maison du sage est maintenant l'univers tout entier. Or qu'est-ce qu'une maison qui n'a pas de dehors, ou qui n'est pas une façon d'être dedans dehors? Pour Levinas, telle n'est pas la véritable nature de l'expérience exilique, et c'est pour cette raison qu'il dénoncera aussi bien l'universalisme abstrait qui fait du chez soi la terre tout entière, que l'idolâtrie du terroir. Dans la mesure où l'existence est une habitation, l'exil forcé conduit dans un non-lieu, dans une non-communauté, et c'est à partir de ce sens social de l'exil que Baudelaire peut décrire dans Le Cygne une telle déshumanisation:

Je pense à la négresse, amaigrie et phtisique,

Piétinant dans la boue, et cherchant l'œil hagard, 
Les cocotiers absents de la superbe Afrique

Derrière la muraille immense du brouillard ${ }^{1}$.

Pour Emmanuel Levinas, l'exil n'est pas le passage d'un monde à un autre, mais il est ce qui rend le monde inhumain, inhabitable; il est l'expérience d'un non-monde. Dans cette perspective, ses analyses sur l'exil du moi ne vont pas non plus reprendre la voie ouverte par Heidegger : l'exil ne sera pas compris à partir de l'être-là, et le monde n'est pas ce qui est maintenu ouvert, mais selon une autre modalité, dans l'exil. Dire comme Heidegger dans Bâtir Habiter Penser, "C'est seulement lorsque ce séjour caractérise déjà la condition humaine que les choses auprès desquelles nous sommes peuvent cependant ne rien nous dire, ne plus nous toucher $»^{2}$, cela revient à manquer la radicalité du non-séjour de l'exil et la dimension sociale de l'habitation. En conséquence, cela revient également à passer à côté de la seconde forme de l'exil, qui n'est pas un autre nom pour la transcendance du Dasein, dans la mesure où il est cette apostasie, cet éveil d'une conscience au-delà du monde, au-delà du possible. Or il est absolument nécessaire d'avoir pu penser l'exil comme une perte radicale du monde pour pouvoir le comprendre comme une tâche reçue par-delà le monde. Seul un être qui peut perdre son monde peut également être passible par amour, peut être l'hospitalité comme recueillement d'une maison ouverte. Il reste à montrer comment, dans les deux volumes d'inédits, Emmanuel Levinas parvient à décrire la délocalisation de l'existence en montrant que seul un sujet qui est chez soi peut être hors de soi dans le non-monde de l'exil subi et dans le non-lieu de l'élection. Il s'agit ainsi de donner toute une signification descriptive au vocabulaire de l'exil, car c'est à partir d'elle que la dimension politique de l'exil peut vraiment être comprise.

Pour une phénoménologie de l'extériorité qui élucide le sens de la transcendance, l'exil n'est pas une simple image un peu excessive pour dire l'être hors de soi, mais une situation très concrète de l'existence, de l'être-jeté dans le monde, qu'il s'agit de comprendre à partir de l'être au-delà de soi. Bien sûr l'exil politique dans ses multiples figures est une donnée première qui appartient au drame de l'existence humaine, mais Emmanuel Levinas ne détourne ce terme du vocabulaire politique et religieux que pour mettre en lumière la signification originaire de l'existence exilique

1. C. Baudelaire, Les fleurs du mal, Paris, Cerf, 2008, p. 192. On peut noter que Totalité et Infini se termine par une citation de Baudelaire pour dénoncer l'existence héroïque insulaire comme vie pour elle-même qui transforme l'identité en une obsession. Le sage stoïcien ne fait pas autre chose que triompher de «l'ennui, fruit de la morne incuriosité qui prend les proportions de l'immortalité» (LXXVI, Spleen, p. 160-161).

2. M. Heidegger, Essais et conférences, trad. fr. A. Préau, Paris, Gallimard, 1958, p. 188. 
à partir de laquelle les formes politiques et religieuses de l'exil pourront recevoir tout leur poids. Sans refuser bien évidemment l'idée traditionnelle d'un exil intérieur face à la violence du monde, qui prend elle-même des significations très diverses, c'est la signification même de l'exil intérieur qui se trouve au cœur de la réflexion en montrant que l'intériorité peut aussi être le lieu d'une expérience de l'au-delà. L'exil est alors le cœur de cette phénoménologie de l'au-delà de la lumière qui se radicalise dans les inédits, puisqu'il est à la fois cette décomposition de toutes les images du monde, mais également la dissolution de tout sens, qui ne conduisent pas à l'absurde, mais à l'essence intime de toute chose, comme dirait Baudelaire dans La Charogne. Il s'agit d'accéder à cette phénoménalité non pas de l'inapparent, mais du sous-jacent, de cette vie qui excède la lumière de l'être. L'exil serait alors ce mouvement de transcendance, qui ne demeure pas un moment de l'immanence, et qui serait cet accès à cette phénoménalité de la pure verbalité et socialité du sens.

\section{Du monde détruit au monde cassé}

Levinas est un remarquable lecteur de Husserl, même s'il a reconnu luimême avoir lu Husserl à partir de Heidegger, et même s'il n'a pas eu accès à toutes les éditions dont disposent aujourd'hui les recherches husserliennes. Or cette proximité entre Husserl et Levinas n'est pas seulement liée à la très grande qualité scientifique des travaux de Levinas, mais elle tient plus profondément encore au partage d'une même question. Il est certes incontestable que Levinas s'éloigne du sujet constituant l'objectivité qui selon lui ne peut pas donner accès à la phénoménalité véritable, cependant il n'en demeure pas moins que de ses premiers travaux sur Husserl jusqu'aux Carnets de captivité il est attentif à la même expérience que celle qui se trouve au cœur de l'idéalisme transcendantal husserlien. De ce point de vue, même s'il a été possible de dénoncer l'intellectualisme de Husserl, il n'y a jamais de philosophie purement théorique qui n'éluciderait pas une expérience originaire, ou alors un tel discours uniquement théorique usurpe le nom de philosophie.

En effet, Levinas prête une attention toute particulière au $₫ 49$ des Idées I sur la conscience absolue comme résidu de la Weltvernichtung, de l'anéantissement du monde. Notamment, dans Théorie de l'intuition dans la phénoménologie de Husserl, Levinas explique parfaitement que le $\$ 49$ ne vise pas à une dépréciation du monde extérieur, mais au contraire doit permettre d'élucider le «sens» de l'existence phénoménale du monde. Ainsi pour saisir la signification pleine et entière de la réduction phénoménologique, il importe de ne pas considérer la dissolution du monde 
comme une simple hypothèse théorique, comme une fiction méthodique, et il est nécessaire d'y voir une expérience fondamentale sans laquelle la reconduction de la réalité au sens, et du sens à l'activité de la subjectivité, n'est pas envisageable. La possibilité d'une conscience sans monde ne résulte pas d'un processus d'abstraction qui maintiendrait la conscience dans une dimension relative (elle demeurerait ce qui a été abstrait de quelque chose) et Levinas commente:

La destruction du monde est un phénomène ayant un sens positif et impliquant nécessairement une conscience, laquelle, par conséquent, une fois le monde détruit, continuerait à avoir une signification transcendante, ne fût-ce que celle du «monde détruit $»^{3}$.

Avec le «monde détruit», Husserl décrit une expérience dans laquelle plus rien d'identique ne se confirmerait de façon continue à la conscience. Husserl met en lumière un conflit d'intentionnalités par lequel il n'y aurait plus que des simulacres et non des objets constants, même relativement. Il est en effet tout à fait pensable que toute cohérence disparaisse de l'expérience, qu'elle ne s'unifie plus: aucun sens ne se dégagerait, aucune synthèse ne se donnerait. La conscience pourrait n'être plus qu'un ensemble désarticulé d'esquisses. Dans toute cette analyse de la perte du monde, il y a bien évidemment un héritage cartésien et un héritage humien à partir desquels Husserl veut lui aussi montrer que la perte possible du sens du monde interdit toute tentation de passer de façon somnambulique du descriptif au normatif, de l'observation du monde au devoir. Pour le dire autrement et pour souligner qu'il ne s'agit pas d'un simple problème théorique, la possibilité d'un effondrement du monde donne à comprendre que le devoir ne peut pas prendre sa source dans les relations mondaines. Ainsi la possibilité de la perte du sens du monde ne conduit pas nécessairement à un scepticisme gnoséologique ou éthique, mais peut conduire à l'évidence originaire condition de toutes les autres.

Or, même si le monde devient dépourvu de sens, même s'il n'est plus qu'un non-monde, cela ne porte pas atteinte à la pure donnée de moimême: je suis simplement le «je» d'un flou de perceptions d'où aucune unité ne se dégage. Levinas sera toujours très attentif à ce qu'il nomme cette étrange structure du «je pur», sa transcendance dans l'immanence, sa veille éternelle, comme étant cette forme d'extra-territorialité par rapport à laquelle le monde se donne soit comme sensé, soit comme détruit. Cela dit, l'échappement du monde est aussi ce qui révèle la conscience absolue

3. Théorie de l'intuition dans la phénoménologie de Husserl, 4 éd., Paris, Vrin, 1978, p. 81. 
et ce qui fait quitter l'attitude naturelle; cette expérience donne accès à la réflexion transcendantale, car elle permet de reconduire réflexivement tout ce qui est donné aux visées de sens et aux modes de l'intuition.

Bien des années plus tard, en 1975, dans Trois notes sur la positivité et la transcendance, Levinas reviendra sur cette expérience et soulignera que la dernière phrase du $₫ 49$ des Idées I est ce qui assure la suppression de la chose en soi et montre que la réalité du monde est purement intentionnelle ${ }^{4}$. Ce radicalisme qui a tant troublé les élèves de Husserl et qui a suscité tant de projets d'un retour à un certain réalisme par-delà l'idéalisme transcendantal absolu ${ }^{5}$ est bien ce qui intéresse Levinas et il pose la question: «Peut-on d'ailleurs s'imaginer véritablement ce que serait concrètement un monde $s^{\prime}$ invertissant en non monde ? ${ }^{6}$. Levinas ajoute que ce qui était difficilement pensable en 1913 l'est beaucoup moins soixante-dix ans plus tard:

Mais sans doute les soixante-dix ans qui nous séparent du texte husserlien - deux guerres mondiales, totalitarisme de droite et de gauche, les massacres, les génocides et l'holocauste - ont-ils signifié - si on peut encore parler ici de sens - une expérience mise en lambeaux et qu'il est impossible de recoller, et cet échec du «je pense» que Kant appelait unité de l'aperception transcendantale - s'efforçant de rassembler en monde les images fantastiques du réel. Échec vécu non pas comme démenti opposé à une audace philosophique, mais comme une catastrophe cosmique, comme celle que nomme le Psaume 82, 5: «Tous les fondements de la terre sont ébranlés»

L'échec auquel pense Levinas n'est plus une modalité de l'être-au-monde, et ce monde déshumanisé et inhabitable n'est plus un monde; cet échec

4. E. Husserl, Idées directrices pour une phénoménologie, trad. fr. P. Ricœur, Paris, Gallimard, 1950, p. 164: «D'autre part l'ensemble du monde spatio-temporel dans lequel l'homme et le moi humain viennent s'insérer à titre de réalités individuelles subordonnées, a en vertu de son sens un être purement intentionnel; il a par conséquent le sens purement secondaire, relatif, d'un être pour une conscience. C'est un être que la conscience pose dans ses propres expériences et qui par principe n'est accessible à l'intuition et n'est déterminable que comme ce qui demeure identique dans le divers motivé des apparences - un être qui au-delà de cette identité est un Rien».

5. Sans vouloir établir une liste exhaustive, on peut citer les noms de Max Scheler, Alexander Pfänder, Adolf Reinach, Moritz Geiger, Roman Ingarden, Edith Stein.

6. Positivité et transcendance, Paris, PUF, 2000, p. 40.

7. Ibid., p. 40-41. Husserl a également pris conscience de la dimension sociale et historique de l'effondrement du monde. Il a longuement médité cette fin d'un monde que fut la Première Guerre mondiale à partir d'une mise en évidence de l'historicité du sens. La crise des sciences décrit également comment la perte de l'Idée de science conduit à un monde vidé de tout sens téléologique véritable et à une déresponsabilisation du sujet. Voir nos études «Historicité et fragilité du sens selon Husserl», in Les débris du sens, P. Hummel et F. Gabriel (éd.), Paris, Philologicum, 2008, et «Husserl et l'impératif de l'Europe idéale», Cahiers de philosophie de l'Université de Caen, nº 47, 2010, Le phénomène Europe, p. 41-60. 
n'est pas la simple mise en cause de mon espace familier, mais il est plus radicalement la rupture même de l'être-au-monde. Dans cette expérience en lambeaux, le vide ne donne rien à voir. D’une façon plus générale, la possibilité que l'expérience se dissipe en simulacres appartient bien à toute subjectivité, et Levinas, même s'il ne fait pas la même analyse de la crise intellectuelle que Husserl, partage la même radicalité dans le refus de tout réalisme dans la compréhension du sens. Cela dit cette expérience de la dissolution du monde n'est pas ici ce qui conduit à l'idée que face au rien l'homme fait face à son propre néant et peut saisir sa tâche d'être. Levinas ne fait pas ici référence à l'angoisse devant le monde étrange et inquiétant, mais à l'expérience même de la perte du monde qui conduit à accomplir la réduction en ce qu'elle est l'épreuve d'une donation de sens qui échoue et qui n'est pas le lieu d'une individuation. Cette expérience met en lumière qu'il n'y a pas d'ipséité toujours déjà là, pas de monde toujours déjà là, mais seulement un «je» qui résiste à la dissolution comme sujet hors-sujet. Elle met en lumière que nous ne sommes pas toujours déjà chez nous, sur un sol stable et immuable, et que nous nous réveillons dans un monde qui nous apparaît comme étranger et que notre «chez soi» véritable est au-delà de ce monde ébranlé.

Ce détour permet de mieux comprendre comment Levinas va penser philosophiquement l'expérience de l'exil lié à la captivité: pour lui également l'expérience du monde cassé de la captivité est aussi ce qui assure la réduction de la réalité au sens. Il n'est pas question bien sûr de minimiser la déréliction propre à l'exil en terre étrangère qui fait perdre quelque chose de sa verticalité, et encore une fois rien n'est plus étranger à Levinas que cette idée stoïcienne selon laquelle le sage est partout chez lui ou bien que la conception romantique de l'exil comme voyage. Le philosophe de l'évasion ne veut pas, en ce qui concerne la captivité, aller contre l'expérience ${ }^{8}$.

Il y a donc bien dans cette description du monde cassé la mise en lumière d'une expérience humienne, dans la mesure où la perte du sens du monde bloque toute tentation de vouloir passer naïvement du descriptif au normatif, de l'observation du monde au devoir. En effet, l'expérience du monde cassé est également cette réduction éthique qui suspend, pour parler comme Hume, «tous les systèmes vulgaires de la morale " ${ }^{9}$ et donne à comprendre que le devoir ne prend sa source ni dans les relations mondaines, ni dans la seule écoute de la raison. L'origine des valeurs ne se trouve pas dans la

8. Il souligne dans les Carnets de captivité que la captivité peut durer au-delà de l'enfermement effectif.

9. Traité de la nature humaine, trad. fr. A. Leroy, Paris, Aubier, 1968, p. 585. 
relation au monde ou dans la pure relation à soi, et c'est une autre relation qui est le lieu originaire de l'évidence morale. Ainsi Levinas s'attache bien à décrire le plus précisément possible le caractère spécifique du vécu qui donne lieu à un devoir.

Ce que Levinas nomme le "monde cassé $»^{10}$ n'est plus exactement une dissolution du monde, mais se comprend plutôt comme un renversement des valeurs pacifiques que sont la propriété, la santé et le respect. Or une telle perte ferait apparaître la «morale absolue», celle qu'on ne peut pas perdre sans se perdre soi-même et qui serait donc le ciment de l'identité: " concevoir la possibilité d'un retour de la paix et la responsabilité à l'égard de cette paix ${ }^{11}$. Le plus important ici est de mettre en lumière que cette expérience d'un monde cassé ne reconduit pas aux actes de la subjectivité isolée: dans cette situation concrète d'existence, dans laquelle s'effectue une neutralisation spontanée du monde comme sol absolu, le monde se révèle relatif au "pour nous» qui est la source du sens. En effet, c'est bien à partir de cette expérience que le monde peut ne pas pouvoir s'unifier que le sens de la réduction devient compréhensible pour l'homme, même s'il est clair que Levinas n'a pas attendu la captivité pour penser la réduction. Dès lors il est bien évidemment essentiel que l'épreuve du monde cassé de la captivité soit l'expérience fondatrice du judaïsme qui dans les Carnets de captivité et autres inédits n'est pas d'abord un phénomène historique, mais est une catégorie, autrement dit une structure du sens ${ }^{12}$. Il y a là

10. Gabriel Marcel a écrit en 1933 une pièce intitulée Le monde cassé (Paris, Desclée de Brouwer) dans laquelle il décrit une vie dissolue, dans laquelle la communication est impossible, qui parvient à se reconstruire par l'accueil de la grâce. L'auteur présente lui-même son ouvrage p. 9: «Le drame amène les deux héros à un point où ils s'apparaissent à eux-mêmes comme engagés dans une réalité qui les transcende infiniment et qu'ils ne sauraient avoir la prétention de dominer». L'héroïne Christiane dit p. 44: «Tu n'as pas quelque fois l'impression que nous vivons... si ça peut s'appeler vivre... dans un monde cassé? Oui, cassé comme une montre cassée. Le ressort ne fonctionne plus. En apparence, il n'y a rien de changé. Tout est bien en place. Mais si on porte la montre à son oreille... on n'entend plus rien. Tu comprends, le monde, ce que nous appelons le monde, le monde des hommes... autrefois il devait avoir un cœur. Mais on dirait qu'il a cessé de battre». Ce thème est très présent dans l'existentialisme chrétien, et là c'est le mystère de la substitution qui permet au monde de retrouver sa vie. Voir ce qu'en dit H.U. von Balthasar, La Dramatique divine, I, Prolégomènes, trad. fr. A. Monchoux, Paris - Namur, Lethielleux - Culture et vérité, 1984, p. 333.

11. E. Levinas, Carnets de captivité, suivi de Écrits sur la captivité et Notes philosophiques diverses (= Euvres 1), R. Calin et C. Chalier (éd.), Paris, Grasset - IMEC, 2009, p. 189.

12. Ibid., p. 75: «J. comme catégorie»; p. 213: «Qu'est-ce donc en fin de compte le judaïsme - en quoi diffère-t-il d'autres religions pleines, elles aussi, d'enseignements moraux et de préceptes de bien - ayant elles aussi, accédé à l'idée de l'unité du principe divin, qu'est le judaïsme sinon l'expérience depuis Isaïe, depuis Job de ce retournement possible - avant l'espoir, au fond de la désespérance - de la douleur en bonheur; la découverte dans la souffrance même des signes de l'élection". 
une toute nouvelle intelligibilité du monde qui reconduit le sens à son origine sociale, au "pour autrui» du sens. L'expérience de la captivité est donc autre que l'expérience de la dissolution du monde en simulacres, car elle donne accès à autre chose qu'à la dimension solipsiste du sens ${ }^{13}$. En effet, il importe de distinguer sans les opposer «débâcle», autrement dit le monde qui se défait, et "captivité», comme suspension qui dure, qui s’installe. Quoi qu'il en soit, débâcle et captivité donnent à voir autre chose que l'anéantissement du monde décrit par Husserl. Husserl mettait en évidence la possibilité pour le monde perceptif de se dissoudre, de ne pas se donner comme monde, alors que Levinas décrit la possibilité pour un monde de basculer dans le non-sens, de se vider de son sens, de cesser d'être monde.

En conséquence, l'expérience du monde cassé ne reconduit pas au sujet autonome constituant l'objectivité tout en se constituant lui-même, mais elle donne à comprendre cette compossibilité de la liberté et de l'être par un autre. Le sujet husserlien, en dépit de sa transcendance dans l'immanence, demeurait encore trop captif du monde et ne parvenait pas à cette expérience de la rupture de la totalité, de la discontinuité de l'histoire, qui rend possible la véritable compréhension du sens. Le sens n'est pas d'abord historique et l'histoire n'est pas le sens de l'être. En étudiant ainsi la corrélation entre l'expérience du monde cassé et l'expérience de l'extériorité, Emmanuel Levinas ne cherche pas à mettre une condition de possibilité qui aurait quelque chose de contingent. Même si tout homme peut au moins une fois dans sa vie faire l'expérience du monde cassé, il s'agit avant tout pour Levinas de montrer en quoi dans sa genèse philosophique personnelle l'expérience du monde cassé a emporté le "je» qui était lié à ce monde, ce qui lui permet la découverte des voies vers l'extériorité et donc l'élucidation d'une expérience de l'au-delà dans les volumes 1 et 2 des Euvres complètes. L'exil n'est pas que le nom emphatique de l'expérience de l'étranger et il est d'abord dans sa forme très concrète la perte de son monde natal, et c'est à partir de là que l'expérience pourra être celle du son, de la métaphore, de l'amour, du visage. Emmanuel Levinas explore d'une façon très ouverte les différentes figures de l'expérience de la transcendance, notamment du désir à l'amour, pour montrer en quoi l'expérience de l'étranger n'est pas une capacité a priori de l'expérience du propre, ce qui constitue le congé le plus radical donné aux analyses des Méditations cartésiennes.

13. Sur cette question, voir D. Franck, L'un-pour-l'autre. Levinas et la signification, Paris, PUF, 2008. 


\section{L'évasion comme accès au sens}

La question de l'exil ne cesse de se confirmer dans la pensée de Levinas et culminera dans Autrement qu'être ou au-delà de l'essence avec la description de l'être en soi comme en exil ${ }^{14}$. Elle s'enracine dans une compréhension très nouvelle de la spatialité par rapport à la conception husserlienne du «je» comme point-zéro de l'espace. Déjà en 1935, dans De l'évasion, se trouvait mis en lumière le besoin d'excendance constitutif de l'homme: "Aussi l'évasion est-elle le besoin de sortir de soi-même, c'est-à-dire de briser l'enchaînement le plus radical, le plus irrésistible, le fait que le moi est soi-même ${ }^{15}$. Ainsi très tôt Emmanuel Levinas considère un moi qui n'est plus dans la répétition de l'identité, dans ce piétinement qui enferme dans une place du monde, fut-elle centrale ${ }^{16}$. Le projet de Levinas est de penser un véritable exode, une rupture avec soi, et non une simple inversion de l'égologie (dans laquelle je ne serai plus qu'un être relatif, au sens transcendantal). Néanmoins, quelles sont les conditions de possibilité d'une telle évasion? Qu'est-ce qui vient briser le rêve d'une auto-élucidation transcendantale?

Ce rêve se finit sans doute avec ce "monde cassé» évoqué dès les premières pages de De l'existence à l'existant:

Des expressions comme «monde cassé» ou «monde bouleversé », pour courantes et banales qu'elles soient devenues, n'en expriment pas moins un sentiment authentique. La divergence entre les événements et l'ordre rationnel, l'impénétrabilité réciproque des esprits opaques comme la matière, la multiplication des logiques, absurdes les unes pour les autres, l'impossibilité pour le moi de rejoindre le toi, et, par conséquent, l'inaptitude de l'intelligence à ce qui devait être sa fonction essentielle - autant de constatations qui, dans le crépuscule d'un monde, réveillent l'antique obsession de la fin du monde ${ }^{17}$.

Un tel monde est un monde qui remet en cause les pouvoirs constituants et reconstituants de l'ego et donc encore une fois la compréhension du sens. En brisant le rêve d'une compréhension purement solipsiste du sens, Levinas dévoile une nouvelle intelligibilité dans laquelle la sensibilité spirituelle,

14. "Le terme en récurrence sera recherché ici par - ou avant - la conscience et son jeu, pardelà ou en deçà de l'être qu'elle thématise, hors l'être et, dès lors, en soi comme en exil» (Autrement qu'être ou au-delà de l'essence, $3^{\mathrm{e}}$ éd., Dordrecht, Martinus Nijhoff, 1986, p. 130).

15. De l'évasion, Paris, Librairie générale française (Le Livre de poche; Biblio-Essais), 1998, p. 98.

16. Ibid., p. 99: «L'évasion, au contraire, met en question précisément cette prétendue paix avec soi, puisqu'elle aspire à briser l'enchaînement du moi à soi. C'est l'être même, le "soi-même", qu'elle fait et nullement sa limitation".

17. De l'existence à l'existant, $2^{\mathrm{e}}$ éd., Paris, Vrin, 1986, p. 25. 
que Husserl a déjà pu envisager dans les Idées II, ne présuppose plus cette fois l'antériorité du «je». Si Levinas reconnaît que Husserl a libéré d'une conception subjective ou formelle du sens, comme de toute conception naturaliste, en montrant que le sens n'est ni dans le sujet, ni dans la chose, mais dans ce quelque chose d'identique qui se donne par exemple dans la perception, il veut donner à voir une tout autre vie du sens dans laquelle le sens ne vient pas d'abord de l'activité du sujet, mais de l'exil lui-même, qui est un autre nom de la proximité. En cela, réfléchir sur la captivité, la situation la plus concrète qui soit, c'est également pour Levinas accéder à une autre compréhension de la vérité: ni la relation au monde, ni la relation à soi ne peuvent fonder l'évidence, et c'est cette autre relation qu'est l'exil qui donne lieu à l'évidence originaire condition de toutes les autres évidences.

Les Carnets de captivité et autres inédits élucident le sens d'une transcendance qui ne se laisse pas ramener à l'immanence:

La connaissance est une pure immanence. Liberté à l'égard du monde, ne pas se commettre avec le monde. Mais elle est moi. Asservissement de l'existence. Le fait même du corps. D'où nécessité de l'évasion, d'une vraie sortie de soi, d'une transcendance ${ }^{18}$.

Cette évasion de l'être est alors la liberté reprise par rapport à une histoire comprise comme processus intentionnel ou inintentionnel, car cette compréhension de l'histoire demeure trop liée à la vérité comme dévoilement ${ }^{19}$. Paradoxalement la captivité est une possibilité d'échapper au primat de l'histoire et de ne plus voir l'intériorité lui être sacrifiée: «Vaincre l'histoire - mais vaincre ce qu'il y a d'être et d'éternité dans l'histoire - non pas par le recours à l'éternité, mais à l'évasion ${ }^{20}$. Levinas ajoute dans les Notes philosophiques diverses:

Le sens n'est pas simplement une finalité. Car la fin est une fin du sens. À la fois mouvement \{incessant\} et orientation - responsabilité d'Autrui - voilà le sens. Ce n'est pas ce qu'on entend par «sens de l'histoire» ${ }^{21}$.

18. Carnets de captivité, p. 56.

19. Voir E. Levinas, "L'Écrit et l'Oral», in Parole et Silence et autres conférences inédites au Collège philosophique (= Euvres 2), R. Calin et C. Chalier (éd.), Paris, Grasset - IMEC, 2011, p. 215: «La vérité comme dévoilement est calquée sur la vision, se retrouve dans l'attitude philologique. L'introduction de l'histoire comme condition de la vérité, ne met pas fin à la pensée comme vision mais la promeut». Levinas précisera dans Totalité et Infini : «Le réel ne doit pas seulement être déterminé dans son objectivité historique, mais aussi à partir du secret qui interrompt la continuité du temps historique» (Totalité et Infini, Paris, Librairie générale française (Le Livre de poche; Biblio-Essais), 1990, p. 51).

20. Carnets de captivité, p. 59.

21. Notes philosophiques diverses, in Euvres 1, p. 303. 
Il s'agit donc bien pour Emmanuel Levinas de prendre aussi congé de l'historialité du Dasein comme assurant le maintien de soi, car un tel maintien trop faible rend également impensable une vraie transcendance qui n'est pas que la fidélité envers le soi-même propre.

Dans cette évasion, il ne s'agit pas d'être en un autre lieu sur terre, par exemple d'être là où se trouve l'autre dont je me préoccupe, mais de se libérer de l'idée d'une place sur terre ou dans l'histoire. L'existant est au-delà de toute identité fixe et Levinas évoque cette «dialectique du je qui s'affranchit de son intimité. L'intimité avec autrui ${ }^{22}$. La sexualité serait alors l'origine du social, c'est-à-dire ce qui donne à voir le phénomène d'abord inapparent du social, à savoir la dimension relationnelle du sens qui donne à penser une solitude ne relevant plus de l'idéalisme subjectif. La captivité qui vient briser le cours de l'histoire est alors révélante de l'existence comme «ce fait d'être absolument voué, de ne pas se pouvoir soustraire ${ }^{23}$. Dans cette vocation il en va d'autre chose que du «devenir hégélien, bergsonien et heideggérien " ${ }^{24}$, car le devenir n'est plus l'accomplissement d'une essence toujours déjà là : «Accomplissement = évasion. Évasion dans quelque chose qui n'est pas être. Félicité» ${ }^{25}$.

Par rapport au bourgeois comme catégorie de l'homme installé qui «ne peut se soustraire au sérieux de sa vie ${ }^{26}$, car il est tout entier sa place dans l'espace social, tout en regardant ce qui est au-delà de sa place comme un simple spectateur, l'exil de la captivité est non seulement ce qui arrache à «ce coin de Bretagne ou de Corrèze», mais également ce qui rend possible une réduction phénoménologique en apprenant «la différence entre avoir et être ${ }^{27}$. Levinas poursuit: "Nous avons appris le peu d'espace et le peu de choses qu'il faut pour vivre. Nous avons appris la liberté $»^{28}$. Il ne faut pas se méprendre sur le sens de ce témoignage philosophique qui n'est en

22. Carnets de captivité, p. 66. Levinas ajoute: «Non pas qu'il y ait là “fusion avec autrui” - précisément il y a dualité du je. Et cette dualité sera décrite précisément dans la concupiscence charnelle - que l'on prend à tort pour un désir comme un autre. La sexualité comme origine du social. Parce qu'il a "intimité" du sexuel, il y a le phénomène du social qui est plus que la "somme des individus" ".

23. Ibid., p. 61.

24. Ibid., p. 134.

25. Ibid., p. 175.

26. Captivité, in Euvres 1, p. 202.

27. Ibid., p. 203.

28. Ibid. Dans un sens à la fois proche et très différent, Shmuel Trigano peut écrire : «Le moins-être du déracinement a révélé ainsi un plus-être. Ce pourrait être là une définition de l'exil» (Le temps de l'exil, Paris, Payot \& Rivages (Rivages poches), 2005, p. 48). Il ajoute que l'exilé n'est pas un égaré quand il découvre une terre intérieure: "Avec l'exil, la terre est devenue une dimension intérieure, plus grande et englobante que l'extériorité. C'est désormais l'intériorité qui assigne sa place à l'extériorité et non plus l'inverse» (p. 100). 
aucune façon un éloge de la captivité et dont le but est d'abord de mettre en évidence qu'il n'y a de possibilité de la liberté que par une libération de soi qui suppose une expérience de la dépossession: au-delà des pesanteurs mondaines l'homme peut assumer le poids de son existence elle-même. En conséquence, Levinas ne veut plus décrire la volonté comme un "se vouloir soi-même» et montre que la volonté elle-même est une telle sortie de soi: «La volonté suppose ce décollement à l'égard de soi. Vouloir, c'est se voir du dehors ${ }^{29}$. Néanmoins il convient de ne pas psychologiser, ni de mettre trop de pathos dans cette sortie de soi pour la considérer dans une certaine neutralité: «Sortir de soi ne signifie pas une apothéose quelconque, un dépassement de sa condition ou l'éclatement de sa définition, mais la possibilité d'une relation avec autre chose que soi-même $»^{30}$.

Levinas, dans les Notes philosophiques diverses, évoque Ulysse qui s'attache au mât pour prévenir la folie liée à la sortie de soi dans l'écoute du chant des sirènes, mais ce n'est pas pour retenir la définition de la philosophie comme retour dans sa patrie ${ }^{31}$. Elle est bien au contraire une marche sans retour qui n'enferme pas dans le statique lié au primat de l'idée de l'être: «Marcher sans se retourner - tel serait le caractère propre de l'idée de l'Infini, marche sans arrêt ${ }^{32}$. Entre Ulysse et Abraham, il s'agit bien pour Levinas de distinguer deux styles d'existence qui ne sont pas conciliables: d'un côté le retour au chez soi à partir de la perte du sol natal, de l'autre la conscience d'être dès la naissance en exil, de ne pas être chez soi, et de la nécessité d'aller au-delà de ce monde sans retour possible. Dans les deux cas le tragique de la vocation n'a pas du tout le même sens ${ }^{33}$.

Levinas effectue donc un renversement de l'ordre qui est totalement assumé: «Le rapport à l'extérieur - le rapport moral - est la condition de la liberté ${ }^{34}$, sans proposer pour autant un idéalisme inversé. En effet, sortir de soi revient à avancer sans éclaireur; cela conduit également à renverser Heidegger, Hegel et Sartre, pour donner à voir une tout autre temporalité issue de l'évasion, dans laquelle je suis ouvert à ce que je ne me suis pas donné. L'exil comme vocation de l'existence consiste bien à revenir à ce qui ne peut être dit en termes d'expérience, dans la mesure où l'expérience suppose toujours une appréhension. L'exil n'est donc pas ici un existential,

29. Notes philosophiques diverses, p. 262.

30. Ibid., p. 291.

31. Voir ibid., p. 344.

32. Ibid., p. 354 .

33. Voir les analyses de C. Chalier, «Emmanuel Levinas "J'ai ouvert... Il avait disparu" (Cantique des cantiques 5, 6)», in Expérience philosophique et expérience mystique, P. Capelle (éd.), Paris, Cerf, 2005, p. 260.

34. Notes philosophiques diverses, p. 396. 
il n'est pas un mode de l'être-au-monde, mais il se comprend comme une ouverture à ce qui n'est pas phénomène, à ce qui vient d'ailleurs que ce qui se donne sur l'horizon du monde. Si le monde détruit de Husserl donne à voir la donation de sens comme vie de l'esprit, le monde cassé de Levinas donne à voir l'évasion comme la vie originaire du sens.

Parole et Silence et autres conférences inédites au Collège philosophique reprend et précise ces thèmes: "Son en soi [au sujet] devient un pour autrui " ${ }^{35}$. En effet, l'oubli de soi qui était déjà très largement mis en valeur s'interprète maintenant comme un "pour autrui» qui devient l'événement de la signifiance. Dans ce désencapsulement du sens, le sens va lui-même être compris comme éthique, et ce tournant éthique correspond bien à une nécessité intérieure de la pensée de Levinas. Si Levinas a très tôt décrit l'arrachement à la quiétude d'une intériorité close, ce n'est qu'avec Totalité et Infini que le thème du «pour autrui » devient crucial. En effet, à partir de l'expérience du monde cassé, la mise en lumière de l'élection est ce qui fait passer du «être pris par autre chose que soi» à l'«être requis par un autre»: «son ipséité réside dans son élection ${ }^{36}$. Bien évidemment il est toujours possible d'effectuer une double lecture d'une telle évolution: d'un côté elle explicite une intention déjà là, et d'un autre côté elle peut sembler un rétrécissement de la problématique de l'exil avec le primat d'autrui. Quoi qu'il en soit, il est nécessaire pour élucider cette ipséité qui est exil et vocation de mieux comprendre la signification de la territorialité.

\section{Le chez soi et l'exil}

La question de l'exil est bien sûr indissociable de celle de l'habitation, de la façon dont l'homme habite la terre, s'y trouve, se tient en elle. Emmanuel Levinas, dans une page célèbre de Totalité et Infini, contre Heidegger et sa pensée du lieu, et d'une façon plus générale contre toute la philosophie occidentale, défend l'idée que la relation à autrui ne se joue pas nécessairement «dans la destinée des peuples sédentaires ${ }^{37}$. Il est indispensable de mettre entre parenthèses toute idolâtrie de la nature et toute idéologie qui identifie l'homme à une place fixe à partir de laquelle la relation à autrui peut avoir lieu, pour retrouver un nomadisme de l'existence qui n'est ni une errance, ni un vagabondage. Exister ne revient pas à se fixer sur son propre projet, mais est l'acte de s'individuer dans la contrainte de répondre à l'appel d'autrui qui m'envoie au-delà de ce que j'imaginais comme mes

35. "Parole et Silence», in Euvres 2, p. 89.

36. «Les Enseignements», ibid., p. 185.

37. Totalité et Infini, p. 37. 
possibles. L'exil est alors cette spatialisation discontinue, asymétrique et vivante dans laquelle l'existence trouve son sens propre.

Il est possible de trouver dans les Carnets de captivité toute une réflexion sur la terre comme sol originaire qui n'est pas sans rappeler les analyses de Husserl : «Sur terre. La terre - point de chute. La terre appui. Être = poids, mais intérieurement connu, dans sa signification existentielle ${ }^{38}$. Ainsi la relation à la terre précède toute relation à l'objet, puisque c'est à partir d'elle qu'il est possible de distinguer l'ici et le là. Dans un passage important des Notes philosophiques diverses, Levinas ajoute: "C'est l'immanence - le fait de rester ici qui est l'événement même du pour soi, condition de la jouissance. Philosophie transcendantale au sens fort: la terre est la condition par excellence ${ }^{39}$. Dans ces pages qui préparent directement Totalité et Infini, la relation à la terre est la condition de toute intention et de tout projet. Ce souci d'une mise en lumière d'une spatialité originaire ne va pas sans la critique du moi substantiel qui bloque tout accès à la véritable nature du chez soi :

La philosophie contemporaine réagissant contre les excès substantialistes de la philosophie classique, tend à enlever au Je sa substantialité et sa suffisance. L'intentionnalité chez Husserl dont le Je n'est qu'un pôle, l'In-der-Welt-sein ou le Miteinandersein de Heidegger, le Je-Tu de Marcel ou de Buber dénoncent à leur façon le Je séparé. Coupé de ce qui la transcende, Monde ou Autrui, la subjectivité ne serait qu'une chose ${ }^{40}$.

Bien évidemment, il n'est pas question pour un penseur de la séparation de refuser l'idée même de substantialité du moi, car cela conduirait à dissoudre le moi dans l'indéfini des relations, et Levinas va élucider la jouissance comme une substantialisation et indépendance du soi. Pour cela il est nécessaire de montrer que le sujet n'est pas d'abord dans l'espace, mais qu'il se spatialise, et c'est bien l'origine de cette spatialisation que Levinas veut interpréter de façon nouvelle. Encore une fois, un «je» qui serait une pure relation perdrait toute substantialité, toute identité massive, et, en sombrant ainsi dans l'apeiron, rendrait impensable la séparation propre au moi. L'intentionnalité husserlienne, la transcendance heideggérienne, le Je-Tu de Marcel Buber, dénoncent justement l'abstraction d'un je coupé du monde, mais demeurent dépendants de ce qu'ils dénoncent en manquant la consistance du moi sans laquelle être requis par un autre est impensable. Il en va là du concept de monde: le monde n'est pas une collection d'objets,

38. Carnets de captivité, p. 52.

39. Notes philosophiques diverses, p. 244-245.

40. "La Séparation», in Euvres 2, p. 274. 
mais il n'est pas non plus l'horizon de Husserl, ou le système de renvois décrit par Heidegger. Le monde de la jouissance est plus originaire que celui de l'usage ou que celui de l'activité constituante, car il est ce qui me nourrit, et son élucidation permet de ne pas faire de "vivre» un verbe intransitif.

Parole et Silence et autres conférences inédites au Collège philosophique accentue encore le vocabulaire de la territorialité (terre, chez soi, domicile, habitation) et ce vocabulaire est ce qui permet d'éviter le vocabulaire des philosophies de la conscience qui réduisent le chez soi à la pure réflexivité et qui manquent le lien eidétique entre le sujet et la terre. Dans des analyses très largement reprises dans Totalité et Infini, Levinas montre comment le sujet ne baigne pas seulement dans la substance du monde, mais l'accueille à distance depuis son chez soi : "Pouvoir accueillir le monde tout en faisant partie du monde, c'est être chez soi ${ }^{41}$. En conséquence, "le pays, la maison, le coin, le lieu» sont "l'être même du moi, ce qui permet seulement l'opposition du moi au non-moi: se fermer dans le monde sur le monde, pour s'ouvrir sur le monde. Il faut ces murs pour qu'il y ait ces portes et ces fenêtres. Parce que le moi est chez soi il peut recevoir et posséder ${ }^{42}$. Ainsi, contre la pseudo-évidence d'un sujet sans lieu, Levinas décrit la situation première de l'existence: dans mon domicile, chez moi, je suis en possession de moi-même: "Je me tiens sur terre - ma suprême condition. Il me suffit comme à Antée de toucher la terre - de me poser - pour y retrouver toute ma puissance ${ }^{43}$. Sans anticiper sur les longues analyses de Totalité et Infini sur le chez soi, l'habitation, la demeure, il faut souligner que la terre est ici plus intime au moi que le moi lui-même, parce qu'elle lui donne de révéler sa puissance. Elle n'est pas la cause occasionnelle du moi, mais ce qui lui donne d'être moi : seul un être qui est chez soi peut être un soi et s'ouvrir à l'absolument autre. Le pour soi du besoin est ce qui fait que le sujet est chez soi dans un monde à prendre. Autrement dit, le moi capable de jouissance est la condition de la phénoménalité du monde. En effet, un être qui serait d'emblée dehors, auprès du monde, se chercherait dans le monde en un vagabondage indéfini, mais, obsédé par la quête de son identité, il ne verrait rien, ni le monde, ni autrui.

Emmanuel Levinas rompt ici avec toute une tradition en refusant de définir le moi pur par la liberté comme c'est le cas, selon lui, de Descartes aux philosophies de l'existence, car c'est à partir de l'intériorité de la jouissance,

41. «Les Nourritures», ibid., p. 166.

42. Ibid. Sur cette question du lieu, voir J.-L. Chrétien, «Lieu et non-lieu dans la pensée d'Emmanuel Levinas», in Emmanuel Levinas et les territoires de la pensée, D. Cohen-Levinas et B. Clément (dir.), Paris, PUF (Épiméthée), 2007, p. 121-138.

43. «Les Enseignements», p. 179. 
et notamment dans sa forme humaine d'être à la fois fermée et ouverte, qu'il peut y avoir un visage, c'est-à-dire l'évidence condition de toutes les évidences. C'est à partir du moi de la jouissance que l'autre n'est pas pour moi une chose sans visage, et dans cette critique de la raison éthique qui se met en place, il s'agit bien de revenir à l'expérience originaire du sens, à ce sens qui se donne à lui-même hors de tout système et qui est autrui lui-même. Ni Husserl, ni Heidegger ne sont parvenus à montrer qu'un monde sensé est d'abord un monde où il y a autrui et que la liberté est la réponse à l'appel d'autrui.

Néanmoins, Levinas prend soin de préciser que cette description du chez soi de la jouissance dans lequel l'existence est prise n'a rien de commun avec le pathos de la littérature de terroir qui manque l'apparaître d'autrui, car l'autre n'est pas un phénomène immédiatement apparent. Le retour à la terre peut libérer des pesanteurs mondaines, mais il n'est en aucun cas un décentrement du sujet, et de ce point de vue il manque la patience de l'altérité: «Il faut un long circuit de pensée pour arriver à la subjectivité de l'autre, pour penser autrui comme un moi hors de moi, ce qui n'est pas une donnée immédiate de la conscience ${ }^{44}$. En deçà de toute idée de nation, il s'agit d'accéder à une expérience originaire du lieu habité, de la maison qui abrite, expérience qui est antérieure à tout projet de soi et à tout souci. L'épreuve du monde cassé présuppose elle-même l'expérience du chez soi: «Même l'étrangeté hallucinante d'un monde étranger ou hostile où le moi semble se défaire, repose sur cette sécurité fondamentale, sur ce repos, dans un lieu, dans une maison, dans un monde ${ }^{45}$. Levinas ajoute: "L'habitation peut devenir cet espace hostile où l'âme est exilée » ${ }^{46}$. Dans la mesure où l'habitation est d'abord le chez soi et la patrie, elle peut être également l'exil. Cette idée simple que l'exil n'a de sens que par rapport au chez soi prend ici une dimension très nouvelle: dans la captivité se donne à voir l'étrange identité du lieu et du non-lieu.

Cette analyse du chez soi et de l'exil dans les inédits est ce qui doit permettre de rompre avec toute philosophie de la totalité. C'est en interrogeant l'expérience du monde cassé qu'il devient impossible de continuer à comprendre la personne comme un moment d'un devenir qui l'englobe. Levinas prépare ainsi son projet de rompre avec la philosophie de Platon à Heidegger, pour mettre en lumière une autre philosophie de l'esprit. Si Husserl a pu montrer que l'esprit est sensible, Levinas, lui, décrit par la

44. «Les Enseignements», p. 182. Sur la littérature de terroir, voir p. 181. Voir également la critique du culte de la terre dans certaines formes du catholicisme dans les Carnets de captivité, p. 183.

45. «Le Vouloir», in Euvres 2, p. 242.

46. Ibid. 
jouissance une sensibilité non intentionnelle, car il ne s'agit pas de jouir d'un objet, mais d'une pure qualité sensible. Or décrire la sensibilité dans sa signification première avant le savoir va précisément être le projet de Totalité et Infini. Il s'agit rien moins que de retrouver la signification verbale du moi qui peut dire le chez soi de la jouissance jusque dans l'exil dans un monde hostile, comme elle peut dire le don, la fécondité: «Jouissance sans savoir. Strictement subjective. Moi» ${ }^{47}$.

Emmanuel Levinas peut alors décrire le moi comme responsabilité qui n'est ni pure pitié, ni pure obéissance, mais qui est fécondité:

[...] il convient que le Moi s'affirme dans la responsabilité, laquelle à la fois est pour... et devant... Elle n'est pas pure pitié parce qu'elle est devant Autrui. Elle n'est pas pure obéissance, car elle est responsable de celui-là même auprès de qui elle est responsable ${ }^{4}$.

Tel est le «moi se détachant de soi - apostasie» ${ }^{49}$. Dans ces textes préparatoires à Totalité et Infini se manifeste très clairement ce détournement du vocabulaire théologique: "Termes religieux qui ne nous servent que de description ${ }^{50}$. Décrire l'apostasie du moi revient à dévoiler tout autre chose qu'une conversion à l'extériorité, puisqu'à partir du chez soi il est possible au plus intime de soi d'être pris par un dehors, d'être arraché à son lieu. Dans Totalité et Infini Levinas va systématiser l'usage des termes religieux (athéisme, religion, apologie, révélation, justice, eschatologie, etc.) pour échapper à l'alternative d'une philosophie de l'intériorisation ou d'une philosophie de l'extériorisation, et pour pouvoir ainsi décrire une vocation qui est une élection et donc le sens social de l'humanité. Emmanuel Levinas a été dans cette méthode marqué par l'usage de l'étymologie propre à Heidegger, et c'est pourquoi il cherche d'une certaine façon à remonter au sens objectif et technique du mot de façon à ce que la signification concrète, matérielle, d'un terme reconduise à une signification bien plus forte que celle qu'il pouvait avoir dans son usage habituel.

Il s'agit bien d'une inversion délibérée de toute la philosophie qui se trouve proposée par Emmanuel Levinas avec le «Ignore-toi toi-même " ${ }^{51}$ des Notes philosophiques diverses. Or il ne s'agit pas simplement d'inverser le "Connais-toi toi-même», mais de repenser la signification de la connaissance de soi. Dire que l'auto-élucidation de l'ego transcendantal

47. Notes philosophiques diverses, p. 245. Comme Levinas le montrera dans Autrement qu'être ou au-delà de l'essence, la jouissance ne s'oppose pas au don, mais en est la condition.

48. Ibid., p. 251.

49. Ibid., p. 266.

50. "Le Vouloir», p. 243.

51. Notes philosophiques diverses, p. 279. 
n'est pas la source ultime du sens ne conduit pas seulement à reconnaitre qu'on se connaît également à partir des autres, mais surtout à remettre en cause la compréhension de la vie du sens et donc du sens de la vie, en accédant à un «je» hors d'être dont la vérité n'est pas celle de l'être, n'est pas celle d'une présence constante qui ne peut ni naître, ni mourir. « Ignoretoi toi-même» n'est pas ici une invitation à ignorer le moi empirique ${ }^{52}$, mais à prendre congé du «je» transcendantal comme unique être absolu, comme origine de tout sens, comme mesure ultime du monde. Or une telle ignorance se gagne par rapport à l'impatience qui est l'essence même du désir. Encore une fois, seul un être qui est chez lui, dans la solide identité de la jouissance, peut chercher à être dans l'ignorance de sa place dans le monde comme condition pour être dans sa tâche reçue et se connaître à partir d'elle: «Élu - moi libéré de soi, puisque pour un autre »53. Le moi n'est pas donné à lui-même dans un redoublement réflexif qui le fait spectateur de lui-même ou à partir de son être-au-monde, mais il est d'abord le moi de la jouissance, qui se découvre dans la rencontre d'autrui. Il ne s'agit donc pas avec l'impératif «Ignore-toi toi-même» de l'oubli de soi comme condition d'une écoute désintéressée, mais bien d'un oubli du soi qui fait apparaître une identité dans une absolue non-coïncidence.

Encore une fois, c'est à partir de la transcendance d'autrui que la liberté est possible et non l'inverse. En effet, la relation éthique comme percée vers l'extériorité présuppose un privilège d'autrui incompatible avec le «Connais-toi toi-même». Comme le développera Totalité et Infini, autrui est l'intelligible premier et non pas moi, et c'est parce qu'il me met en question que je peux découvrir ma liberté, notamment dans sa dimension destructrice. Autrement dit autrui n'est pas la simple occasion d'une prise de conscience de moi dans une relation intersubjective, et la liberté que je découvre n'est pas issue du savoir que je peux avoir sur moi-même par réflexion. Le «Ignore-toi toi-même» est ce retrait en soi, comme dit Levinas ailleurs, cette systole de la conscience, comme mouvement originaire fondamental qui rend possible la liberté.

Un tel oubli du soi ne doit pas être pensé ici comme un acte vertueux et la description ne doit pas être dupe de la morale, selon la recommandation du début de Totalité et Infini. Il est hors de question d'écrire une éthique énonçant les grandes règles de l'agir, même s'il s'agit bien d'une éthique qui met en lumière des valeurs, qui énonce des commandements, qui donne à comprendre le bien et le mal à partir du "pour autrui ». On peut souligner que déjà le «Connais-toi toi-même» n'était pas une règle empirique de

52. Ce que Levinas envisage dans Autrement qu'être ou au-delà de l'essence.

53. Notes philosophiques diverses, p. 281. 
sagesse, pas plus que ce n'était le principe d'une technique du bonheur ou de la liberté, mais cette fois avec le «Ignore-toi toi-même» il s'agit de rompre avec l'éthique des valeurs absolues qui paradoxalement bloque l'accès à une véritable pensée de la valeur comme nourriture de la volonté. Levinas cherche bien à prendre congé de l'éthique comme soumission de sa décision à la mesure d'une décision idéale et irrévocable, et avec les inédits il inaugure une éthique du tâtonnement dans laquelle j'apprends de l'autre ce qui est bon pour lui, ce qui est proprement s'ignorer. Ainsi, le «Ignore-toi toi-même» n'est pas un nouveau commandement qui renverserait simplement l'impératif de la connaissance de soi, mais c'est une nouvelle façon de comprendre le sens d'un commandement. La véritable relation éthique conduit à ne plus trouver en soi, dans l'auto-élucidation du « je» transcendantal, les normes absolues de la connaissance et de l'action, pour saisir ces normes à partir des situations concrètes dans lesquelles je suis engagé dans l'existence. Entendre le "Ignore-toi toi-même» depuis l'épreuve sans distance d'autrui, c'est accomplir l'exigence du retour aux choses mêmes en s'affranchissant d'un humanisme universaliste dans lequel l'homme est considéré comme une essence et non comme une personne ${ }^{54}$. C'est autrui qui m'ordonne, m'assigne, de m'ignorer, et on comprend que seul le vocabulaire théologique peut décrire ce qui est ainsi absolument propre aux relations de personnes et qui échappe toujours à la langue de la conscience objectivante. Dans cette toute nouvelle compréhension de l'éthique, le «pour autrui» de l'élection est ce qui fait que le sens de l'existence comme vocation est possible. Mon unicité qui ne provient pas d'une différence ne tient ni à ma place dans le monde, ni à ma capacité de réflexion, mais à ce "pour un autre" antérieur à toute résolution. L'ignorance ne consiste donc pas, pour reprendre l'expression de Ricœur, à être soi-même comme un autre, car dans l'élection le soi est hors de l'être. Ainsi la réponse d'Emmanuel Levinas au monde cassé ne se trouve pas dans l'instauration de nouvelles valeurs ${ }^{55}$, mais dans un retour sur la vie du sens et sa dimension sociale qui fait que dans la paternité et la filialité je suis investi de mon être ${ }^{56}$, et c'est pourquoi «le moi est la discontinuité même» ${ }^{57}$.

54. Voir Totalité et Infini, p. 88

55. Le visage n'est pas une valeur, car une valeur, qu'elle soit relative ou absolue, est un objet de la volonté et est toujours lié à un jugement, et c'est pourquoi il rend possible une autre forme d'obligation que celle fondée sur des valeurs. L'évidence du visage est la condition de toutes les valeurs, ce qui fait la signifiance véritable des valeurs, et l'éthique comme philosophie première ne consiste bien évidemment pas à simplement mettre l'éthique à la première place, mais modifie le sens même d'une philosophie des valeurs.

56. Voir Notes philosophiques diverses, p. 282.

57. Ibid., p. 319 . 
Il est alors possible à Levinas d'opposer la dimension solipsiste du moi et la dimension sociale du moi qui s'accomplit dans le don: «la relation avec autrui n'a pas la structure abstraite de la coexistence - mais est le fait de donner ${ }^{58}$. Il s'agit certes de mettre fin à la théorie abstraite de l'Einfühlung, de l'empathie ${ }^{59}$, en poursuivant, après Husserl et Heidegger, la mise en lumière de son non-sens phénoménologique, mais plus largement encore il s'agit de prendre congé de toute pensée de l'intersubjectivité qui par principe manque l'antériorité du "pour autrui». En effet, le don n'est pas ce qui succède à l'expérience d'autrui comme alter ego, mais il est le "pour autrui» lui-même: autrui est reçu dans le don. Levinas insiste sur l'idée que même dans sa signification transcendantale l'Einfühlung manque la transcendance d'autrui, car dans la nouvelle compréhension de la relation avec autrui qu'il veut développer il ne s'agit en aucun cas d'un transport en l'autre, dans la mesure où cette relation n'est plus liée à la saisie d'une essence commune et à une identification de soi et de l'autre. Cela dit, la question de l'empathie est peut-être encore davantage l'occasion de s'éloigner de toute l'analyse de l'être-avec dans Être et Temps, et notamment par la distinction de deux dimensions du moi: «Moi comme identification à travers le temps et moi comme des-identification $=$ fécondité ${ }^{6}{ }^{6}$. Dans ces passages qui anticipent une nouvelle fois les analyses de Totalité et Infini, la fécondité est elle-même un terme descriptif qui, permettant d'éviter le vocabulaire occultant de la conscience, parvient à mettre en lumière que «la volonté n'est pas Entschlossenheit, mais bonté» ${ }^{61}$. Dans cette nouvelle perspective, qui s'oppose aux analyses heideggériennes de l'ouverture et de la résolution, penser, c'est se surprendre à partir de ce qui de moi est avant moi, c'est briser le monde étroit de l'horizon a priori des possibles: «Recevoir et donner - caractéristiques ontologiques du Moi, en dehors de la passivité et de l'activité ${ }^{62}$. Telle est la pointe de la critique de Levinas: le «je» transcendantal et le Dasein ne peuvent pas donner, car ils ne peuvent

58. Notes philosophiques diverses, p. 387. Passant du descriptif au prescriptif, Levinas écrira dans Totalité et Infini: «Il faut que je sache donner ce que je possède» (p. 185).

59. La position de Levinas sur l'empathie est en fait plus nuancée, car si l'éthique est une optique spirituelle, elle consiste à apprendre à considérer autrement l'empathie à partir d'une conception renouvelée de la sensibilité. Il s'agit aussi d'une certaine façon de sauver l'empathie contre Husserl et Heidegger. Voir Notes philosophiques diverses, p. 387: «Dès lors la relation avec autrui n'a pas la structure abstraite de la coexistence - mais est le fait de donner. Autrui n'est pas pour moi - l'alter ego - c'est le pauvre. La fin de la théorie de l'Einfuhlung comme d'une sympathie. Ou plutôt la sympathie - c'est la sympathie avec le pauvre».

60. Notes philosophiques diverses, p. 389.

61. Ibid., p. 406.

62. Ibid., p. 437. 
pas recevoir. Il s'agit là du retournement radical du projet d'une maîtrise héroïque de soi qui masque le sens de la vie, c'est-à-dire la signification sociale de la vocation ${ }^{63}$.

Tout homme disait Husserl, reprenant Descartes, doit au moins une fois dans sa vie découvrir son «je» transcendantal, mais c'est à un tout autre éveil auquel veut donner accès Levinas avec cette nouvelle intelligibilité du moi comme don et comme patience qui est «la naissance latente d'une conscience qui est déjà au-delà du possible» ${ }^{64}$. $\mathrm{Moi}^{65}$ se fait patience « dont l'ultime fondement est une responsabilité infinie devant l'infini de l'autre» ${ }^{66}$. À propos de cette patience qui est bonté, Emmanuel Levinas ajoute dans cette conférence du 27 janvier 1959 au Collège philosophique et intitulée "Au-delà du possible»: "Nous le montrerons un jour " ${ }^{67}$. Néanmoins, avec cette volonté qui s'ouvre à l'au-delà du possible, et qui est de ce point de vue tout autre chose qu'un désir de l'impossible, c'est-à-dire le désir de transformer l'impossible en possible, Emmanuel Levinas montre que le moi est une des-identification et une délocalisation, et donc un exil, et qu'exister, c'est rompre avec la maîtrise pour se faire patience d'élu. Ainsi, en donnant à comprendre que ni le «je pur» selon Husserl, ni le Dasein d'Être et Temps, ne possèdent une identité assez consistante pour pouvoir subir l'exil violent de la perte du monde, pas plus qu'ils ne peuvent vivre l'exil de transcendance dans l'acte de recevoir et de donner, Emmanuel Levinas ouvre une nouvelle voie dans la compréhension de l'existence comme ouverture à l'au-delà du possible dans une responsabilité où le soi est un avenir qu'il ne trace pas lui-même.

Toutes ces analyses font signe bien évidemment vers ce qui sera le cœur de la pensée d'Emmanuel Levinas, autrement dit le centre qui unifie toute son œuvre, à savoir l'élucidation de la substitution dans Autrement qu'être ou au-delà de l'essence. Dans le temps de la patience, contre toute idée d'une égalité à soi, le soi-même est cette altération de l'essence qui est une expulsion de soi hors de soi dans la substitution à l'autre. Dans la patience absolue du "pour autrui », du sacrifice, le soi est sous le poids d'autrui, et se trouve lui-

63. Sur la place du don dans une éthique qui est une philosophie première, il est difficile de ne pas penser au texte de 1953 de Vladimir Jankélévitch Philosophie première: «L'homme peut donner plus que ce qu'il a et, en l'espèce, donner son être; sacrifier, au-delà de l'avoir, l'ipséité propre: ainsi l'aimant, dans l'amour, se dessaisit coûteusement de tout soi-même, et traverse l'instant de la douleur " ( ${ }^{\mathrm{e}}$ éd., Paris, PUF (Quadrige), 2011, p. 191). Sur le rapport de Levinas à Jankélévitch, comme à toute la philosophie française de son temps, le travail de recherche reste à faire.

64. "Au-delà du possible», in Euvres 2, p. 309.

65. Et non «le moi» comme le rappelle très justement Didier Franck.

66. "Au-delà du possible», p. 310.

67. Ibid. 
même dans cet exil compris comme non-coïncidence radicale. Emmanuel Levinas élucide ainsi philosophiquement l'idée biblique que la souffrance et la mort dans leur passivité peuvent être actives. Aimer l'autre plus que sa propre vie est ce qui libère de toute éthique fondée sur la persévérance dans son être. Pouvoir donner sa vie n'est pas une forme limite de la vie éthique, mais ce qui rend toute valeur véritablement vivante, car il n'est possible de donner sa vie pour des valeurs que si on la donne pour autrui. Il était donc nécessaire d'accéder à un moi bien plus substantiel que le «je» transcendantal de Husserl ou que le Dasein de Heidegger pour mettre en lumière la radicalité et la possibilité de la substitution qui est le sens de l'humain.

Entre l'intentionnalité de la conscience, la transcendance du Dasein et la nudité de l'élu la discussion est loin d'être close et il en va de la compréhension même de l'ipséité. Quoi qu'il en soit, l'apport des inédits est de montrer que si l'ipséité a pour fondement la transcendance, la vocation comme exil de transcendance n'est véritablement intelligible qu'à partir de l'exil comme perte qui donne à voir une ouverture à la transcendance avant toute intention et tout projet. Seul un être capable de jouissance, c'est-à-dire qui se réjouit ou s'attriste de ce qui se donne, peut souffrir dans l'exil du monde cassé, mais seul un tel être peut être également capable d'être à partir d'autrui. Partir du moi de la jouissance pour penser ce qu'est l'humain, c'est mettre en évidence que le sens de l'humain ne vient à l'idée que dans cette situation concrète de l'existence qui consiste à recevoir et à donner. La stabilité de la jouissance est ce qui délivre de la résolution comme fidélité envers le soi-même propre et donc du souci d'une autoconstance: dans l'exil de la vocation autrui n'est pas l'occasion de me possibiliser, il est mon avenir en tant que son avenir me temporalise. Ainsi, pour conclure, la philosophie d'Emmanuel Levinas est vraiment en genèse dans ces deux volumes des CEuvres complètes, car il prend définitivement congé du «je» transcendantal qui demeure à l'abri de sa transcendance et du Dasein qui ne peut pas être détruit par le monde, et, au-delà de ces abstractions, il parvient à décrire l'existant qui prend conscience de lui-même dans l'expérience que «Tous les fondements de la terre sont ébranlés» (Psaume 82, 5), car il faut savoir ce que signifie perdre pied pour comprendre que l'expérience de l'étranger est ce qui permet à l'expérience de soi de se constituer, sans être pour autant un nouveau "fondement». Avec ces inédits s'effectue donc un changement de signe, car il ne s'agit pas simplement de prolonger la description du besoin d'excendance de l'homme, mais de se libérer de toute philosophie du fondement pour penser l'élection, même au cœur de la souffrance. Dès lors, il n'est plus question d'intersubjectivité comme fondement ou d'être-avec, mais de la gratuité du don qui est la forme originaire de la relation avec autrui et la lumière d'avant la lumière. 
On peut se demander cependant si pour être soi comme en exil la lumière du monde n'est pas cependant nécessaire. Si la tâche d'être soi n'est pas donnée par le monde, n'est-elle pas toujours reçue dans le monde, c'est-àdire également avec sa dimension de finitude? La patience absolue tient-elle compte de la finitude essentielle de l'existence humaine, de l'action humaine, ou bien ne continue-t-elle pas à proposer au moi un idéal inatteignable? En outre, à chercher toujours plus loin la phénoménalité de l'obscur, de l'invisible, de l'au-delà de la lumière, jusqu'au quasi non-phénomène, la lumière de l'être n'en vient-elle pas à s'éteindre et peut-on encore penser la possibilité de la rencontre? Emmanuel Levinas a montré la possibilité d'aller au-delà de la transcendance de l'ego et de la transcendance du Dasein en tant que ce sont des transcendances encore prises dans l'immanence, et toute l'analyse du monde cassé n'a pas reconduit à la pure immanence de l'exil intérieur, mais à la transcendance de ce qui est au-delà de la lumière de la conscience. De la jouissance à l'amour il a décrit une existence à distance de soi. Comme il le dit déjà dans Le Temps et l'Autre ${ }^{68}$, le pathétique de l'amour est une relation avec ce qui se dérobe à jamais. Néanmoins, quelle est la phénoménalité de ce qui se dérobe à jamais, de l'absolument absent? "Se dérober à jamais », ce n'est plus la réserve de l'être, la pudeur, qui a pu être décrite par Heidegger, mais quelle est ici alors la donnée phénoménologique, puisqu'il ne s'agit même pas de la donnée paradoxale de ce qui se donne dans sa réserve même? Husserl disait lui-même que si l'autre est inaccessible directement il est tout de même là devant moi, en chair et en os, en personne. Comment penser une absence qui n'est pas la forme d'une donnée, d'une présence, d'une incarnation? S’il est au plus haut point important de montrer qu'autrui m'est donné à aimer sans m'être donné à voir, aimer autrui n'est-ce pas aussi le voir dans la lumière de son être sans en briser le secret? Si Levinas est un tournant dans le souci de décrire la transcendance spécifique de Dieu sans lui imposer un mode d'intuition qui viendrait d'ailleurs que de lui et si de cette façon il met en évidence que Dieu n'est ni un objet, ni une Idée, cette volonté d'aller au-delà de l'immanence ne conduit-elle pas au-delà de la phénoménalité? L'inaccessible, l'invisible, l'absent ne se phénoménalisent qu'en se donnant. Vouloir décrire la transcendance de l'absent, vouloir sortir de l'expérience, mettre entre parenthèses l'expérience ${ }^{69}$, pour penser Dieu, n'est-ce pas

68. "Le temps et l'autre», in Cahiers du Collège philosophique. Le choix, le monde, l'existence, J. Wahl (éd.), Paris, Artaud, 1947, p. 184; texte différent dans Le Temps et l'Autre, Paris, PUF (Quadrige), 1994, p. 78.

69. Notes philosophiques diverses, p. 231. À comparer encore avec Vladimir Jankélévitch, Philosophie première, p. 4: «La métempirie n'est donc pas encore une certaine modalité de l'empirie, par exemple une extrême ténuité ou une intensité insupportable du perçu, 
vouloir prononcer les noms de l'origine et vouloir parler et penser comme Dieu lui-même? N'y a-t-il pas là, en dépit de toutes les analyses sur le corps, un oubli de la corporéité?

Emmanuel HousseT

mais elle désigne ce qui est hors de toute expérience possible: car si cet ultrasensible est ce qui excède, en fait, la portée actuelle de nos sensoria, le suprasensible est ce qui ne peut être perçu». 